\title{
CD40L-adjuvanted DNA vaccine carrying EBV-LMP2 antigen enhances anti-tumor effect in NPC transplantation tumor animal
}

\author{
LEI LEI I , JIANHUI LI' ${ }^{1}$ MEIQING LIU ${ }^{2}$ XIAOMING HU⿱2 , YA ZHOU ${ }^{2}$ SHIMING YANG \\ ${ }^{1}$ Department of Otolaryngology, Head and Neck Surgery, Institute of Otolaryngology, Chinese PLA General Hospital, Beijing, China \\ ${ }^{2}$ School of Optoelectronics, Beijing Institute of Technology, Beijing, China
}

\begin{abstract}
CD40L, a costimulatory molecule for dendritic cells (DCs) and B cells, can serve as an adjuvant for enhancing the specific immune response induced by DNA vaccine carrying tumor-associated antigens. In this study, we investigated the potential of CD4OL as an adjuvant to enhance the anti-tumor effect mediated by a DNA vaccine based on the Epstein-Barr virus-latent membrane protein 2 (EBV-LMP2) antigen. The plasmids capable of expressing the fusion protein EBV-LMP2-CD4OL were constructed. Expression vector $p V A X 1$ and plasmid expressing the individual antigen EBV-LMP2 were used as control groups. These plasmids were used to immunize female BALB/c mice (4-6 weeks old) at days 0 , 7 and 14. The results suggest that immunization with DNA vaccines carrying fusion gene EBV-LMP2$C D 40 L$ can induce specific immunity more effectively than the plasmid expression individual antigen $E B V$-LMP2. In order to evaluate the anti-tumor effect of this DNA vaccine, we constructed a tumor bearing mouse model. After immunization, the tumor bearing mouse model, DNA vaccination with EBV-LMP2-CD4OL plasmid significantly inhibited tumor growth in the tumor bearing mouse model and enhanced the tumor inhibition rate. This study demonstrated that encoding the EBV-LMP2 tumor antigen within an EBV-LMP2-CD4OL DNA vaccine generates an effective antitumor response against $E B V$ tumor, which may be a promising method to improve the antitumor immunity of DNA vaccine.
\end{abstract}

Key words: CD4OL, DNA vaccine, Epstein-Barr virus, nasopharyngeal carcinoma, anti-tumor.

(Centr Eur J Immunol 2018; 43 (2): 117-122)

\section{Introduction}

Epstein-Barr virus (EBV) infects most people worldwide. EBV has oncogenic potential and is strongly associated with several lymphomas and carcinomas, including nasopharyngeal carcinoma (NPC), that together total 200,000 cases of cancer each year [1]. Although NPC is highly radiosensitive, it is resistant to these therapies in about $30 \%$ of patients. Therefore, it is necessary to develop novel treatments for NPC [2]. All EBV-associated cancers express viral proteins that allow high selective immunotherapeutic targeting of the malignant cells. A number of therapeutic EBV vaccines have been tested in clinical trials with evidence of immune boosting and clinical responses in NPC patients. EBV infection in NPC cells exhibits a latency II pattern and the viral proteins EBNA1, LMP1 and LMP2 are expressed [3]. LMP2 mRNA transcripts are detected in more than $98 \%$ of NPC biopsies and protein can be detected by immunohistochemistry in $>50 \%$; this discrepancy likely being due to the lower sensitivity of the latter technique [4]. LMP2 contains many CD8+
T-cell epitopes [4-6] and is therefore considered the best CD8+ T-cell target expressed in NPC. A smaller number of CD4 T-cell epitopes have also been identified in LMP2 and there is evidence that these epitopes are displayed by LMP2-positive cells as well [7]. Therefore, LMP2 is an ideal target for NPC immunotherapy.

Cancers are complex and harbor an array of tumor associated antigens, and approaches to target multiple tumor antigens and utilize nucleic acid-based vaccines incorporating costimulatory molecules of the immune system are emerging [8]. The advantage of nucleic acid vaccines is that multiple antigens can be delivered easily with one immunization, do not require prior knowledge unlike peptide vaccines and are not restricted by the patient's HLA type [9]. Furthermore, DNA encoding costimulatory molecules such as CD40L, CD70, and glucocorticoid-induced TNF receptor-related protein (GITR) can be incorporated into vaccines to enhance their immunogenicity $[10,11]$.

CD40L acts on DCs to induce or "license" CD8+ T cell responses $[12,13]$. Studies have demonstrated the efficacy

Correspondence: Lei Lei, PhD, Department of Otolaryngology, Head and Neck Surgery, Institute of Otolaryngology, Chinese PLA General Hospital, 28 Fuxing Road, Beijing 100853, China, e-mail: wiseleilei@qq.com

Submitted: 13.02.2017; Accepted: 8.05.2017 
of exogenous CD40L as a vaccine adjuvant to promote increased $\mathrm{T}$ cell proliferation and effector functions, including $\mathrm{T}$ cell poly-functionality and cytokine production, and to polarize cellular and humoral immune responses towards a Th1 phenotype [13-15].

In the present study, a DNA vaccine (pVAX1-EBVLMP2-CD40L) was generated encoding the murine CD40L extracellular domain fused to the EBV-LMP2. The results suggest that immunization with DNA vaccines carrying fusion gene EBV-LMP2-CD40L can induce specific immunity more effectively than the plasmid expression individual antigen EBV-LMP2. In order to evaluate the anti-tumor effect of this DNA vaccine, we constructed a tumor bearing mouse model. After immunization the tumor bearing mouse model, DNA vaccination with EBV-LMP2CD40L plasmid significantly inhibited tumor growth in the tumor bearing mouse model and enhanced the tumor inhibition rate. This study demonstrated that encoding the EBV-LMP2 tumor antigen within an EBV-LMP2-CD40L DNA vaccine generates an effective antitumor response against EBV tumor, which may be a promising method to improve the antitumor immunity of NPC DNA vaccine.

\section{Material and methods}

\section{Construction of DNA plasmids}

To generate pVAX1-EBV-LMP2-CD40L, DNA encoding amino acids of EBV-LMP2 (Genbank: CAD53382.1) was incorporating the full extracellular domain of CD40L (Genbank: NM_011616.2) linked by (G4S)3 (Fig. 1a). The fusion coding sequence was inserted into plasmid pVAX1 (Invitrogen, USA). The control plasmid, pVAX1-EBV-LMP2, was also constructed.

\section{Flow cytometry analysis}

293 T cells were transfected with DNA plasmids using Lipofectamine $2000^{\mathrm{TM}}$ transfection reagent (Invitrogen, USA). After $48 \mathrm{~h}$ of transfection, cells were collected and centrifuged. After the cells were treated for fixing and permeability with BD Cytofix/Cytoperm ${ }^{\mathrm{TM}}$ Fixation/ Permeabilization Kit, the cells were incubated with fluorescein isothiocyanate (FITC)-labeled anti-LMP2 antibody (Biorbyt, orb8424) and phycoerythrin (PE)-labeled anti-mouse CD40L antibody (Abcam, ab25568) at room temperature for $60 \mathrm{~min}$. After incubation, expression of LMP2 or CD40L in the transfected cells was detected by flow cytometry.

\section{Immunization}

Female Balb/c mice (4-6 weeks old) were purchased from Beijing Weitong Lihua Experimental Animal Technology Co. Ltd. (Beijing, China). Four groups of ten mice were immunized by intramuscular injection followed by electroporation at week 0 , and boosted twice at weeks 1 and 2 . The immunization dose of each mouse was $50 \mu \mathrm{g}$ of plasmid. The immunization program is shown in Figure 1B.

\section{Antibodies responses}

Antibody responses in the immunized mice were identified by ELISA. The microtiter plates were coated with $5 \mu \mathrm{g} / \mathrm{mL}$ recombinant EBV-LMP2 protein in bicarbonate buffer, and incubated overnight at $4{ }^{\circ} \mathrm{C}$. After blocking with $5 \%$ powdered milk in PBS containing Tween-20, sera obtained from mice at 14 days after the last immunization were serially diluted in PBS, added to the plates and incubated for $1 \mathrm{~h}$ at room temperature. After washing with PBS, the plates were incubated with $1: 5000$ dilution of horseradish peroxidase (HRP)-conjugated goat anti-mouse IgG antibody at $37^{\circ} \mathrm{C}$ for $30-45 \mathrm{~min}$. The color was developed at room temperature and was read at $450 \mathrm{~nm}$. The cut off value was set as 2.1 times over negative controls.

\section{ELISPOT assay}

The level of LMP2-specific IFN- $\gamma$-secreting cells in mice was determined using an ELISPOT assay. Mice were sacrificed 2 weeks after the third immunization. Splenocytes were harvested from individual mice. The IFN- $\gamma$ ELISPOT assay was performed according to the protocol supplied by the manufacturer. In brief, 96-well precoated polyvinylidene fluoride plates (Dakewe Biotech Ltd., Shenzhen, China) were coated with anti-mouse interferon (IFN- $\gamma$ ) monoclonal antibody (mAb) overnight at $4^{\circ} \mathrm{C}$, and blocked for $1 \mathrm{~h}$ at $37^{\circ} \mathrm{C}$ before experiments. The purified splenocytes $\left(5 \times 10^{5}\right.$ cells/well $)$ together with EBV-LMP2 protein $(5 \mu \mathrm{g} / \mathrm{mL}$, Biorbyt, orb383147) were added to the well and incubated at $37^{\circ} \mathrm{C}$ for $20 \mathrm{~h}$. Each test condition was performed in triplicate. The spots were counted and analyzed with the ELISPOT reader (AID, Germany).

\section{NPC tumor animal model and tumor immunotherapy studies}

To generate a cell line stably expressing EBV-LMP2, CNE-2 cells were transfected with plasmid pIRES-neoEBV-LMP2 and then subjected to selection by treatment with G418. This cell line is hereafter referred to as CNE2-LMP2. Female BALB/c nude mice (6-8 weeks old) were used in all experiments. Protocols were performed following national and institutional guidance for animal care and were approved by the Chinese PLA General Hospital. All tumor immunotherapy studies included 5 mice per group. A total of $5 \times 10^{5} \mathrm{CNE}-2-\mathrm{LMP} 2$ cells were injected i.h. into the right shoulder back. Mice were then injected i.m. with plasmid DNA on day 3, 10, and 17 into hind quadriceps muscles. Tumor volume was measured 3 times per week using a digital caliper, measuring the longest diameter (a) and shortest width (b). Tumor volume was calculated by the formula $\mathrm{V}\left(\mathrm{mm}^{3}\right)=0.5 \times \mathrm{ab}^{2}$. The tumor inhibition rate was measured according to the following 
A


Fig. 1. Schematic diagram and expression of the DNA vaccines. A) Schematic diagram of the DNA vaccines that contain LMP2 and CD40L. B) Detection of the expression of DNA vaccine in 293T cells. The expression of LMP2 and CD40L protein was examined by flow cytometry

formula (average tumor weight in the control group - average tumor weight in the treatment group)/average tumor weight of the control group $\times 100 \%$ ).

\section{Statistical analysis}

GraphPad Prism 5.0 software was used for all analyses. To compare individual time-points, a one-way ANOVA was used to compare three or more groups. Student's t-test was performed to determine significance between two groups. The Kaplan-Meier method was employed to construct the survival curve. Differences for which $p<0.05$ were considered statistically significant.

\section{Results}

\section{Construction and expression of pVAX1-EBV- LMP2-CD40L}

Eukaryotic expression plasmids were constructed as described in the Methods section (Fig. 1A). In pVAX1EBV-LMP2-CD40L, the coding region of LMP2 was fused to the N-terminal of CD40L. In order to detect the expression of the constructs in a eukaryotic system, $293 \mathrm{~T}$ cells were transfected with these plasmids and the cells were collected and assayed for LMP2 or CD40L by flow cytometry at $48 \mathrm{~h}$ after transfection. As shown in Figure 1B, LMP2 and CD40L was detected in cells transfected with pVAX1-EBV-LMP2-CD40L, whereas nothing could be detected in cells transfected with pVAX1. These results confirmed that the recombinant plasmids can be successfully expressed in the eukaryotic system.

\section{Antibody and ELISPOT assays}

Mice were injected via intramuscularly and electroporation with pVAX1-EBV-LMP2, pVAX1-EBV-LMP2CD40L and pVAX1 (negative control) plasmids, respectively. At week 2 after the final immunization, serum from each mouse was collected and anti-LMP2 was monitored. As shown in Figure 2A, pVAX1-EBV-LMP2, pVAX1EBV-LMP2-CD40L plasmids elicited significant titers of anti-LMP2 after vaccination. Furthermore, the level of specific anti-LMP2 in mice vaccinated with pVAX1-EBVLMP2-CD40L resulted in a significantly higher level than those vaccinated with $\mathrm{pVAX} 1-\mathrm{EBV}-\mathrm{LMP} 2$.

The levels of EBV-LMP2-specific T cells were determined by ELISPOT assay as described in Material and methods. Mice were sacrificed at day 14 after the third immunization. Splenocytes were prepared and stimulated with the recombinant EBV-LMP2 protein. As shown in Figure $2 \mathrm{~B}$, the number of spots detected in the assay using cells from the pVAX1-EBV-LMP2-CD40L vaccinated groups was significantly higher than the number of spots detected using cells from the pVAX1 and pVAX1-EBV-LMP2.

These results indicate that immunization with the CD40L fused gene not only elicits higher titers of anti-EBVLMP2 antibody but also induces higher EBV-LMP2-specific $\mathrm{T}$ cell response, indicating the CD40L fusion gene vaccination might be an effective anti-tumor method. 



Fig. 2. Induction of enhanced LMP2-specific immune responses by CD40L fusion vaccination. DNA vaccine immunization schedule for Balb/c mice. Mice were immunized at weeks 0, 1, and 2. For assessing overall immune response, mice were sacrificed 2 weeks after the final immunization to collect blood and spleen for analysis of humoral and cellular immune responses. A) Mice were immunized with pVAX1-EBV-LMP2 or pVAX1-EBV-LMP2-CD40L at a dose of $50 \mu \mathrm{g}$ DNA delivered via i.m./EP. Mice that received pVAX1 served as negative controls. Two weeks after final vaccination, IgG antibodies specific to LMP2 in sera were detected by ELISA. B) ELISPOT assays were performed to test the ability of T cells to produce IFN- $\gamma$ in specific response to LMP2 protein antigen

\section{EBV-LMP2-CD40L DNA vaccine can significantly inhibit CNE-2-LMP2 tumor growth in mice}

To study the effect of DNA vaccine on tumor growth, tumor-bearing mice were vaccinated with the DNA vaccine. The effect of immunotherapy was evaluated by measuring the tumor size. Three days after tumor cell injection, mice were vaccinated three times with DNA vaccine, and the tumor volume was measured. Tumor growth in mice treated with CD40L-fusion DNA was significantly inhibited compared with that in the $\mathrm{pVAX} 1$ vector group or non-CD40L-fusion DNA $(p<0.05)$ (Fig. 3A). We also measured tumor weight $(p<0.05)$ (Fig. 3B) and calculated the tumor growth inhibition rate of the different groups of mice after vaccine treatment. Tumor growth inhibition rates in mice immunized with CD40L-fusion DNA were significantly higher when compared with mice immunized with the non-CD40L-fusion DNA or pVAX1 (Fig. 3C).

\section{Discussion}

In recent years, immune therapy technology has developed rapidly, and has played an important role in the treatment of many diseases. This is especially true in the treatment of cancer, where immune therapy has shown great potential and therapeutic effect [16]. Anti-tumor immunotherapy using vaccines has become a prominent research topic, and much progress has been made using this approach. In 2012, the world's first anti-tumor therapeutic vaccine, PROVENGE®, was approved by the United States Food and Drug Administration (FDA) for prostate cancer treatment [17]. However, many studies have indicated that a vaccine containing only an antigen is not effective enough to induce an anti-tumor immune response, inhibit tumor growth, inhibit recurrence, or inhibit metastasis $[18,19]$. At present, the synergistic combination of antigens with different immunostimulatory molecules to produce significantly improved anti-tumor vaccines is a key avenue of investigation.

CD40L, a costimulatory molecule belonging to the tumor necrosis factor superfamily (TNFSF) that can stimulate both dendritic cells (DCs) and B cells for enhancing $\mathrm{T}$ cell and antibody $(\mathrm{Ab})$ responses. CD40L activates DCs and enhances the priming of the cytotoxic CD8 T cell response. CD40L also enhances the survival and differentiation of activated $\mathrm{B}$ cells, leading to increased germinal center (GC) formation, immunoglobulin isotype switching, antibody somatic affinity maturation, and the generation of long-lived plasma cells. These immunostimulatory functions of CD40L have made it an attractive vaccine adjuvant [20]. CD40/CD40L interaction may promote the survival of EBV, however, Younesi suggest that Epstein-Barr virus inhibits the stimulatory effect of TLR7/8 and TLR9 agonists but not CD40 ligand in human B lymphocytes [21]. This shows that the expression of CD40L did not affect the 


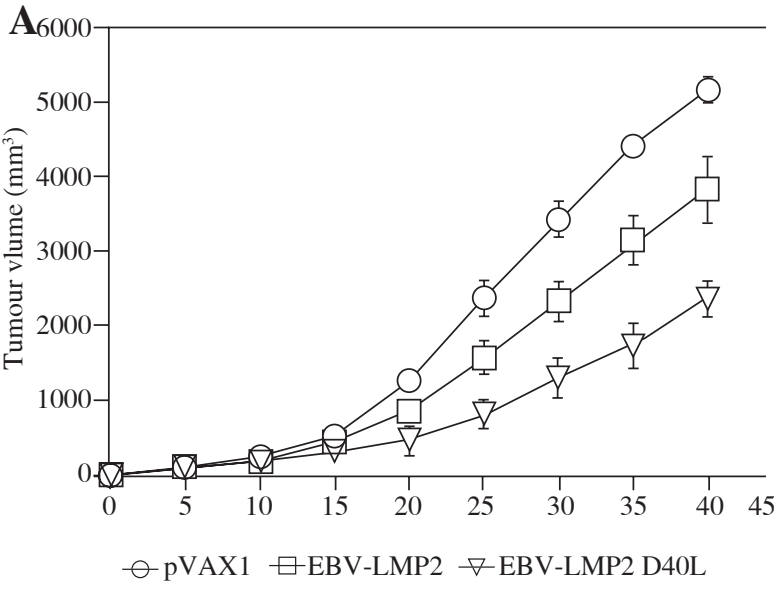

C

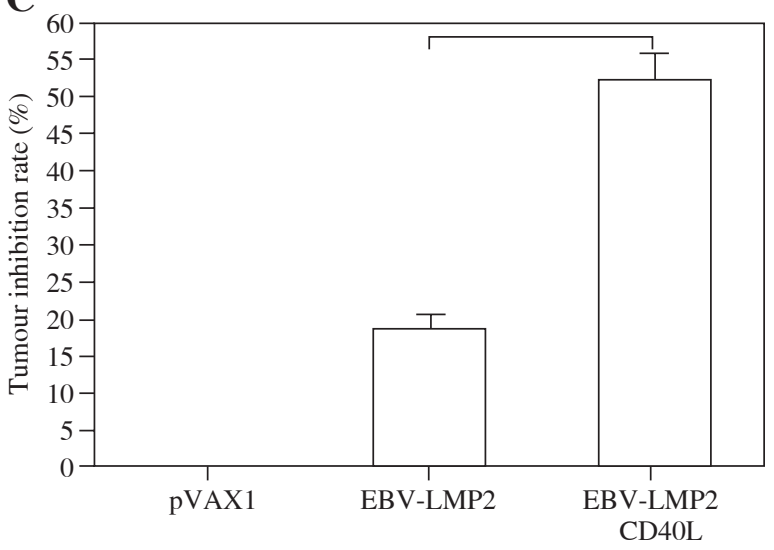

immune response induced by EBV. In addition, Khanna also suggest that an EBV-specific T-cell can be a product for adoptive immunotherapy based on activation-dependent expression of CD154 [22].

So, in this study, in order to improve the immunogenicity and anti-tumor effect of the vaccine, we designed a fusion vaccine with $\mathrm{CD} 40 \mathrm{~L}$ as an intramolecular adjuvant. This study demonstrated that encoding the EBV-LMP2 antigen within an LMP2-CD40L DNA vaccine generates an effective antitumor response against CNE-2-LMP2 tumor. This is mainly due to the fact that CD40L binds to CD40 on antigen presenting cells (APCs), thereby inducing APC activation. APCs, in turn, prime cytotoxic T lymphocytes (CTLs) [23].

\section{Conclusions}

Taken together, our results demonstrate that CD40L- fusion DNA vaccine can significantly improve the anti-tumor effect. It may be mainly due to the fact that CD40L can be an effect of targeting DC in vivo [24], which is advantageous to enhance the efficiency of antigen presentation. Therefore, the ability of vaccine-induced immune response is improved. This method could be used with other tumor vaccine antigens to create a robust specific immune re-
B

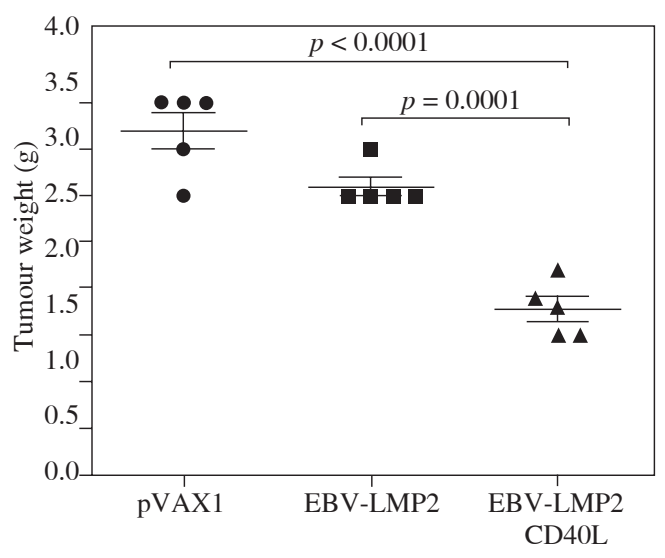

Fig. 3. CD40L-fusion DNA vaccination-induced antigen-specific immunity confers protection against tumor challenge. Three days after being challenged with tumor cells, the mice were immunized three times at an interval of 7 days with the pVAX1-EBV-LMP2 or pVAX1-EBVLMP2-CD40L plasmid, respectively. A) Growth curve of CNE-2-LMP2 tumor. B) Mean tumor weight. C) Tumor inhibition rate

sponse by targeting antigen to DC. Therefore, our study suggests that this model of increasing DNA vaccine immunogenicity through fusion expression of CD40L and antigen is a promising anti-tumor therapy.

In conclusion, this study demonstrated that encoding the EBV-LMP2 antigen within an LMP2-CD40L DNA vaccine generates an effective antitumor response against CNE-2-LMP2 tumor.

The authors declare no conflict of interest.

\section{References}

1. Cohen JI (2015): Epstein-barr virus vaccines. Clin Transl Immunology 4: e32.

2. Hutajulu SH, Kurnianda J, Tan IB, Middeldorp JM (2014): Therapeutic implications of Epstein-Barr virus infection for the treatment of nasopharyngeal carcinoma. Ther Clin Risk Manag 10: 721-736.

3. Lun SW, Cheung ST, Lo KW (2014): Cancer stem-like cells in Epstein-Barr virus-associated nasopharyngeal carcinoma. Chin J Cancer 33: 529-538.

4. Tsang CM, Deng W, Yip YL, et al. (2014): Epstein-Barr virus infection and persistence in nasopharyngeal epithelial cells. Chin J Cancer 33: 549-555. 
5. Ramayanti O, Juwana H, Verkuijlen SA, et al. (2017): Epstein-Barr virus mRNA profiles and viral DNA methylation status in nasopharyngeal brushings from nasopharyngeal carcinoma patients reflect tumor origin. Int J Cancer 140: 149162.

6. Zheng Y, Parsonage G, Zhuang X, et al. (2015): Human Leukocyte Antigen (HLA) A*1101-Restricted Epstein-Barr Virus-Specific T-cell Receptor Gene Transfer to Target Nasopharyngeal Carcinoma. Cancer Immunol Res 3: 1138-1147.

7. Lin X, Gudgeon NH, Hui EP, et al. (2008): CD4 and CD8 T cell responses to tumour-associated Epstein-Barr virus antigens in nasopharyngeal carcinoma patients. Cancer Immunol Immunother 57: 963-975.

8. Subramaniam DS, Liu SV, Giaccone G. (2016): Novel approaches in cancer immunotherapy. Discov Med 21: 267-274.

9. Jorritsma SH, Gowans EJ, Grubor-Bauk B, Wijesundara DK (2016): Delivery methods to increase cellular uptake and immunogenicity of DNA vaccines. Vaccine 34: 5488-5494.

10. Li D, Huang Y, Du Q, et al. (2016): CD40 Ligand and GMCSF Coexpression Enhance the Immune Responses and Protective Efficacy of PCV2 Adenovirus Vaccine. Viral Immunol 29: 148-158.

11. Barr TA, Carlring J, Heath AW (2006): Co-stimulatory agonists as immunological adjuvants. Vaccine 24: 3399-3407.

12. Soliman H, Mediavilla-Varela M, Antonia SJ (2015): A GMCSF and CD40L bystander vaccine is effective in a murine breast cancer model. Breast Cancer (Dove Med Press) 7: 389397.

13. Curran KJ, Seinstra BA, Nikhamin Y, et al. (2015): Enhancing antitumor efficacy of chimeric antigen receptor T cells through constitutive CD40L expression. Mol Ther 23: 769-778.

14. Fabricius D, Breckerbohm L, Vollmer A, et al. (2011): Acute lymphoblastic leukemia cells treated with $\mathrm{CpG}$ oligodeoxynucleotides, IL-4 and CD40 ligand facilitate enhanced anti-leukemic CTL responses. Leukemia 25: 1111-1121.

15. Palumbo RN, Nagarajan L, Wang C. (2011): Recombinant monomeric CD40 ligand for delivering polymer particles to dendritic cells. Biotechnol Prog 27: 830-837.

16. Papaioannou NE, Beniata OV, Vitsos P, et al. (2016): Harnessing the immune system to improve cancer therapy. Ann Transl Med 44: 261.

17. Gulley JL, Mulders P, Albers P, et al. (2015): Perspectives on sipuleucel-T: Its role in the prostate cancer treatment paradigm. Oncoimmunology 5: e1107698.

18. Grunwald T, Ulbert S. (2015): Improvement of DNA vaccination by adjuvants and sophisticated delivery devices: vaccine-platforms for the battle against infectious diseases. Clin Exp Vaccine Res 4: 1-10.

19. Li L, Petrovsky N (2016): Molecular mechanisms for enhanced DNA vaccine immunogenicity. Expert Rev Vaccines 15: 313-329.

20. Kwa S, Lai L, Gangadhara S, et al. (2014): CD40L-adjuvanted DNA/modified vaccinia virus Ankara simian immunodeficiency virus SIV239 vaccineenhances SIV-specific humoral and cellular immunity and improves protection against a heterologous SIVE660 mucosal challenge. J Virol 88: 95799589.

21. Younesi V, Nikzamir H, Yousefi M, et al. (2010): Epstein Barr virus inhibits the stimulatory effect of TLR7/8 and TLR9 agonists but not CD40 ligand in human B lymphocytes. Microbiol Immunol 54: 534-541.

22. Khanna N, Stuehler C, Conrad B, et al. (2011): Generation of a multipathogen-specific T-cell product for adoptive immuno- therapy based on activation-dependent expression of CD154. Blood 118: 1121-1131.

23. Soong RS, Song L, Trieu J, et al. (2014): Direct T cell activation via CD40 ligand generates high avidity CD8+ T cells capable of breaking immunological tolerance for the control of tumors. PLoS One 9: e93162.

24. Auten MW, Huang W, Dai G, Ramsay AJ (2012): CD40 ligand enhances immunogenicity of vector-based vaccines in immunocompetent and CD4+ T cell deficient individuals. Vaccine 30: 2768-2777. 